\title{
Sternocleidomastoid Muscle
}

National Cancer Institute

\section{Source}

National Cancer Institute. Sternocleidomastoid Muscle. NCI Thesaurus. Code C33616.

A superficial neck muscle and a key anatomical landmark, which divides the neck into anterior and lateral regions. It originates on the manubrium and the clavicle, and inserts onto the mastoid process and the superior nuchal line. It functions to flex the neck, move the chin cranially, and assists in elevating the rib cage during inspiration. 